\title{
Impacts of Covid-19/SARS-Cov-2 on Pediatric Population Infected With Asthma and How Did it Impact the Management of the Same
}

\author{
Shivansh Srivastava ${ }^{1}$, Manju Chandankhede ${ }^{2}$ and Dhruba Hari Chandi ${ }^{3}$ \\ ${ }^{1}$ first year MBBS student at Datta Meghe Medical College, Shalinitai Meghe \\ Hospital and research centres, Nagpur, India \\ ${ }^{2}$ HOD Department of Biochemistry Datta Meghe Medical College, Shalinitai \\ Meghe Hospital and Research Centre, Nagpur, India \\ Department of Microbiology Jawaharlal Nehru Medical College, Datta Meghe \\ Institute of Medical Sciences Sawangi (Meghe), Wardha \\ Corresponding author email: Shibu142@gmail.com
}

\begin{abstract}
Wether asthma constitutes a risk factor for coronavirus disease-2019 is unclear. This article aims to assess wether asthma, is associated with higher Covid-19 risk or severity in pediatric age group and provide a review, how did the pandemic impact the management of this chronic disease in the pediatric population. A systematic literature review was carried out on PubMed and Google Scholar under the following head - Covid-19, Paediatric population, Asthma, Management. Out of all the articles found 8 were short listed. Based on which this systematic review has been produced. The COVID19 pandemic has been hovering since 2 years now and still it remains unclear wether it makes the condition of asthma worse specifically in the pediatric age group and the same unmitigated spread created countless difficulties for diagnosis of the same. This article elaborates on the same. There is barely any data available as of now pertaining the same issue therefore, initial concerns about children and young people with asthma being infected with the virus have not been found out or realised. Clearly, our understanding of the relationship between the infection and pediatric asthma still remains explorative. Studies are required beyond counting to lay our conclusions on the same.
\end{abstract}

KEY WORDS: COVID-19, PAEDIATRIC POPULATION, ASTHMA, MANAGEMENT

\section{INTRODUCTION}

(Zhou et al. 2019) The current outbreak of this coronavirus disease-2019, caused by the severe acute respiratory syndrome coronavirus-2 (SARS-CoV-2), started in the end of the year 2019, in a Chinese location Wuhan. (Dhok et al. 2020) It was declared as a global pandemic in March 2020 by the WHO. The SARS-CoV- 2 is the newest member of the corona virus family and impacts the respiratory, nervous and gastrointestinal systems in the individual.

Biosc Biotech Res Comm P-ISSN: 0974-6455 E-ISSN: 2321-4007

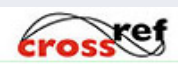

Identifiers and Pagination

Year: 2021 Vol: 14 No (6) Special Issue

Pages: 264-266

This is an open access article under Creative

Commons License Attribn 4.0 Intl (CC-BY). DOI: $h t t p: / / d x$.doi.org/10.21786/bbrc/14.6.55
However, the virus would target the lungs and the nose causing complication like the common cold, bronchitis and pneumonia. The second most frequent symptoms in humans however is common cold.

The virus uses it's spike to attach to human cell membrane this complete process is activated by a specific enzyme by the cell this variant is particularly more infectious because of it's unique spike protein activation by the enzyme Furin found in the host cell. This enzymes is present in many locations in the human body including the lungs, liver and the gastrointestinal tract, hence the virus to attack multiple regions at a particular instance in time. This enzyme also promotes the stability and the transmission of the virus.

On the surface of the human cells the enzyme ACE2 acts as the receptor that enables SARS-CoV-2 to launch it's

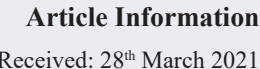

Received: $28^{\text {th }}$ March 2021 ccepted after revision: $29^{\text {th }}$ May 2021 
attack the virus's spike protein binds to the receptor and releases it's RNA within the cell.

(Gawai et al. 2020 Hobbs et al. 2020) Interestingly, atopic children have under-expressed ACE-2 enzyme's receptor in the lungs and it is required for the coronavirus recognition and infection. (Birelliwar et al. 2020) The first case of Covid-19 was reported in Wuhan in January 2020.(Corne et al. 2002) The youngest patient of COVID19 remains a 36 hour old new born Studies suggest that the disease has severe complications in adult population compared to the pediatric one. However, we still lack the evidence to state the effects of asthma on children infected with the COVID-19.

The diagnosis of the pediatric asthma during the pandemic may become complicated since the symptoms overlap along with the symptoms of the Covid-19 Disease. Hence, the line of protocol for the Covid-19 have to be applied to all those children exhibiting symptoms like cough or shortness of breath. Also the follow-up consultations had to be substituted by addressing patients on phone calls and the routine tests like the FS, IOS, BDT, FeNO measurement of AHR tests respectively did not take place amid the pandemic and, very few patients underwent the full diagnosis of asthma. Changes like these have created remarked changes on the medical practice, approach and medication that are used to manage asthma.

\section{DISCUSSION}

The pandemic has resulted in numerous complexities for clinicians, who have had to act and choose an approach based on very limited data available. That is a major cause for concerns raised regarding the pediatric community that is infected by the virus in future. In case of asthma the patient experiences mucus hypersecretion, damaged epithelium and airway obstruction. (Zade et al. 2020) The ICS that are used for the treatment of asthma advocate viral duplication, delay it's clearance and therefore, it impacts the immunity and this created a perfect atmosphere for corona virus to live in.

(Burhani et al. 2020) Another 2002 lancet study states that the people with asthma have an higher risk of showing symptoms that are infected with the common cold virus and, corona virus is the second most common cause for cold in humans. The above mentioned reasons make it necessary for the doctors to include the pediatric asthma under umbrella of high risk factors for sever COVID-19 outcomes. Data from Centres for Disease Control (CDC) of COVID-19 cases illustrates that out of the total $96 \%$ cases where the age was known only $8.1 \%$ were below the age of 18 . However, the mortality rate in the children was below 1\%. Similar logistics were seen in Wuhan, China where less than 1000 individuals were found infected with the virus who all were aged below 16(Zhou et al. 2020). (Choi et al. 2020) Another report suggests that out of 651 children below the age of 19 admitted to hospitals in the UK with the same disease little less than 10 died(these individuals were those who found profound comorbidity).
An analysis of pediatric COVID-19 hospitalisation data from 14 states in the US by CDC found out that the average rate of hospitalisations was low in children compared to adults. (Hasan et al. 2020) It was 8 in a 100,000 for children whereas, for adult it stood at 165 Also, out of every three children hospitalised only 1 required intensive care. Another systematic review of 18 studies including 1065 individuals, mostly pediatric ones with SARS-CoV-2 infected with fever, dry cough and fatigue or were asymptomatic. One individual who was an infant presented with pneumonia was treated under intensive care and they recovered.

Another retrospective study that involved 366 children less that the age of 16 hospitalised for respiratory infections, out of the only 6 were confirmed with Covid19 however once again no one was known to have been infected with asthma According to a report from the WHO-China Joint Mission on COVID-19, the largest scale up to date, 55,924 cases were confirmed by Feb 2020 out of which only $2.4 \%$ were under the age of 19 and out of these $2.5 \%$ were critically ill and only .2\% were in critical conditions however, once again no one was reported to be suffering with asthma. In Malaysia two boys aged 2 and 11 were reported. However all those individuals mentioned above mentioned infected with COVID-19 weren't known to have asthma.

How did the pandemic impact management of the chronic disease of asthma: Diagnosis of the pediatric asthma during COVID-19 may be complicated since the symptoms of COVID-19 and severe asthma are more or less the same. As a result, even if cough history is indicating towards asthma the screening approach applied would be the same as COVID-19 ones. Oral corticosteroids were not suggested to the people in the beginning with covid-19 due to increased risk of immune depression and a even worse viral response. Some state the opposite of this hypothesis. Routine spirometry testing was also suspended. Since the procedure can propose a harm to the health care workers and patients.

European Respiratory society notes that the lung function tests are risky since infection can spread from one individual to the other and through the surrounding surfaces even ahead. And suggests that the full operation of lung function test may only resume as the virus prevalence is low and reliable arrangements are there for its control. Experimental studies have suggested that the ICS and bronchodilator can suppress the corona virus replication and cytokines synthesis. Nebulisation is considered as a potential risk for aerosol generation and the transmission of virus thus if a asthma exacerbation comes up various different approaches are supposed to be taken.

\section{CONCLUSION}

The relation between the chronic disease asthma and COVID-19 in pediatric population remains unclear and studies need to go beyond scope until we land on one specific conclusion. Management of the same had been 
severely impacted due to the upsurge in the cases and spread of virus as a result of which many consultations that are supposed to be happening in person were conducted on mobiles or through the internet. A complete new fashion was also seen in the routine treatments that were utilised to deal with asthma.

\section{REFERENCES}

Birelliwar, A. and Athawale, V., 2020. Angela kapoor, Waqar M. Naqvi. Early gait training program and proprioceptive neuromuscular facilitation in the patient with post debridement amputation-A rare case report. Medical Science, 24(105), pp.3421-3426.

Burhani, T.S. and Naqvi, W.M., 2020. Telehealth--A Boon in the Time of COVID 19 Outbreak. Journal of Evolution of Medical and Dental Sciences, 9(29), pp.2081-2085.

Choi, S.H., Kim, H.W., Kang, J.M., Kim, D.H. and Cho, E.Y., 2020. Epidemiology and clinical features of coronavirus disease 2019 in children. Clinical and experimental pediatrics, 63(4), p.125.

Corne, J.M., Marshall, C., Smith, S., Schreiber, J., Sanderson, G., Holgate, S.T. and Johnston, S.L., 2002. Frequency, severity, and duration of rhinovirus infections in asthmatic and non-asthmatic individuals: a longitudinal cohort study. The Lancet, 359(9309), pp.831-834.

Dhok, A., Butola, L.K., Anjankar, A., Shinde, A.D.R., Kute, P.K. and Jha, R.K., 2020. Role of Vitamins and Minerals in Improving Immunity during Covid-19
Pandemic--A Review. Journal of Evolution of Medical and Dental Sciences, 9(32), pp.2296-2301.

Gawai, J.P., Singh, S., Taksande, V.D., Sebastian, T., Kasturkar, P. and Ankar, R.S., 2020. Critical Review on Impact of COVID 19 and Mental Health.

Hasan, S.S., Capstick, T., Zaidi, S.T.R., Kow, C.S. and Merchant, H.A., 2020. Use of corticosteroids in asthma and COPD patients with or without COVID-19. Respiratory medicine, p. 106045.

Hobbs, C.V., Martin, L.M., Kim, S.S., Kirmse, B.M., Haynie, L., McGraw, S., Byers, P., Taylor, K.G., Patel, M.M., Flannery, B. and COVID, C., 2020. Factors Associated with Positive SARS-CoV-2 Test Results in Outpatient Health Facilities and Emergency Departments Among Children and Adolescents Aged< 18 YearsMississippi, September-November 2020. Morbidity and Mortality Weekly Report, 69(50), p.1925.

Latchoumi, T.P., Ezhilarasi, T.P. and Balamurugan, K., 2019. Bio-inspired weighed quantum particle swarm optimization and smooth support vector machine ensembles for identification of abnormalities in medical data. SN Applied Sciences, 1(10), pp.1-10.

Zade, R., Sahu, P., Shende, G., Phansopkar, P. and Dadgal, R., 2020. Comprehensive physical therapy improves functional recovery in a rare case of stroke associated with asthma: A case report. Medical Science, 24(105), pp.2893-2899.

Zhou, M., Zhang, X. and Qu, J., 2020. Coronavirus disease 2019 (COVID-19): a clinical update. Frontiers of medicine, 14(2), pp.126-135. 